\title{
Diffuse Pulmonary Meningotheliomatosis Diagnosed by Transbronchial Lung Biopsy
}

\author{
Roberto Bernabeu Mora ${ }^{a}$ Juan Miguel Sánchez Nieto ${ }^{a}$ Chunshao Hu \\ Eduardo Alcaraz Mateos ${ }^{\mathrm{b}}$ Alberto Giménez Bascuñana ${ }^{\mathrm{b}}$ \\ Manuel Rodríguez Rodríguez ${ }^{c}$ \\ Divisions of a Pneumology, ${ }^{b}$ Pathology and ${ }^{c}$ Radiology, Hospital Morales Meseguer, University of Murcia, Murcia, Spain
}

\section{Established Facts}

- Minute pulmonary meningothelial-like nodules (MPMNs) are usually unique lesions in the lung parenchyma. They are asymptomatic and are normally detected incidentally during autopsy or the examination of resected lung specimens.

\section{Novel Insights}

- Diffuse pulmonary meningotheliomatosis, which is presented as multiple MPMNs, is less common. We have described a case of diffuse pulmonary meningotheliomatosis detected by computed tomography and diagnosed by transbronchial lung biopsy, which is very unusual.

\section{Key Words}

Pulmonary nodules · Minute pulmonary meningothelial-like nodules · Transbronchial lung biopsy · Computed tomography

\section{Abstract \\ Minute pulmonary meningothelial-like nodules (MPMNs) are usually unique lesions in the lung parenchyma. Diffuse pulmonary meningotheliomatosis, which is presented as multiple MPMNs, has been less frequently described. MPMNs are mainly asymptomatic and are diagnosed after lung sur- gery or during autopsy. We report on a patient with multiple}

and bilateral pulmonary nodules, some of which were cavitated, diagnosed with diffuse pulmonary meningotheliomatosis by transbronchial lung biopsy. Diffuse pulmonary meningotheliomatosis should be included in the differential diagnosis of bilateral lung nodules.

Copyright $\odot 2013$ S. Karger AG, Basel

\section{Introduction}

Minute pulmonary meningothelial-like nodules (MPMNs) are common incidental pathological findings, but the rate of detecting MPMNs varies between 0.07

\section{KARGER}

E-Mail karger@karger.com www.karger.com/res
(C) 2013 S. Karger AG, Basel

0025-7931/13/0862-0145\$38.00/0
Roberto Bernabeu Mora, MD

Division of Pneumology, Hospital Morales Meseguer University of Murcia, Avda Marques de los Velez s/n ES-30008 Murcia (Spain)

E-Mail rbernabeumora@ hotmail.com 
Fig. 1. a CT scan of the chest showing diffuse multiple minute nodules distributed randomly throughout both lungs. b Magnified transaxial image of the lower left lung showing a clearer image of central lucency in several of the micronodules.

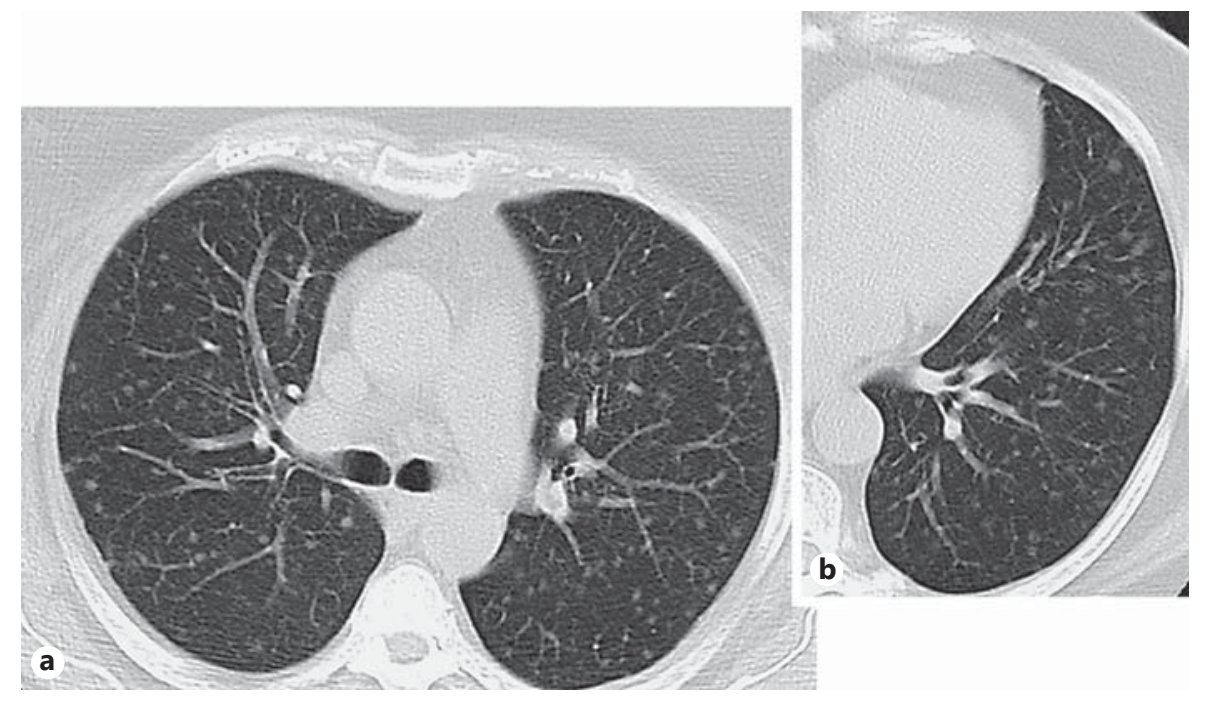

and $13.8 \%$ depending on the source of the lung tissue examined (autopsy or surgical resection specimens) [1-4]. Single lesions were defined as MPMNs, whereas multiple nodules were termed diffuse pulmonary meningotheliomatosis or MPMN-omatosis $[1,5]$.

\section{Case Report}

A 59-year-old woman was referred from the Gastroenterology Department in May 2012, following the observation of multiple lung nodules on a computed tomography (CT) scan of the chest and abdomen performed to investigate chronic diarrhoea. Her past medical history was significant for uterine cancer treated with hysterectomy, double adnexectomy, and radiation therapy, with no evidence of recurrence at follow-up visits. She had also undergone multiple surgical interventions for anal abscesses and had severely weakened sphincter muscles, believed to be the cause of the chronic diarrhoea. She was a smoker of 20 pack-years. Her spirometry results were normal and she reported no respiratory symptoms. The physical examination was completely normal. Her laboratory test values were normal. The patient tested negative for ANA, anti-DNA, ANCA, rheumatoid factor, RNP, Sm, Ro, La, Scl70, and Jo-1. Tumour marker levels were normal. HIV serology was negative, as was a tuberculin skin test. Transthoracic echocardiography and mammography were also normal. A chest CT scan confirmed the presence of multiple disseminated minute nodules distributed randomly throughout both lungs (fig. 1). Most of the nodules were poorly defined and had a cotton wool appearance, and several were cavitated. Bronchoscopy showed a normal mucosa. The analysis of bronchial aspirate and bronchoalveolar lavage fluid specimens showed no tumour cells or microorganisms. The examination of transbronchial biopsy specimens showed interstitial proliferation of epithelioid cells with oval monomorphic nuclei, without atypia or mitosis, arranged in irregular clusters with poorly defined borders. The immunohistochemical staining of the cells was negative for cytokeratin AE1/AE3, chromogranin A, synaptophysin, actin, desmin, and $\mathrm{HMB}-45$, and positive for vimentin, EMA, progesterone receptors, and CD56, leading to a pathological diagnosis of pulmonary meningotheliomatosis (fig. 2). The patient continues to progress favourably and is currently free of symptoms.

\section{Discussion}

Histologically, MPMNs appear as small nests of epithelioid cells in the interstitial tissue of the lung $[2,5]$. The origin and nature of these nodules are unknown. It has been suggested that they might originate from muscle cells rather than meningothelial cells [6] and that steroids might have a role in their growth [7]. Some authors have reported that MPMNs lack mutational damage and suggested that they might represent the transition between reactive and neoplastic proliferation [8]. Others, in turn, have shown that MPMNs and meningiomas have identical cytologic and immunohistochemical features $[2-4,9,10]$. In our patient, immunohistochemical staining was positive for vimentin, progesterone receptor, and EMA, as well as for CD56, a recently described marker [4]. Most MPMNs measure between $100 \mu \mathrm{m}$ and $3 \mathrm{~mm}$ and are randomly distributed throughout the subpleural interstitium of the lungs $[2,11,12]$. They mostly occur in women, aged between 16 and 94 years, and are most common in the 6th decade of life $[4,13]$. In most cases, MPMNs occur as single lesions. Although multiple MPMNs may be detected in lung specimens, this 


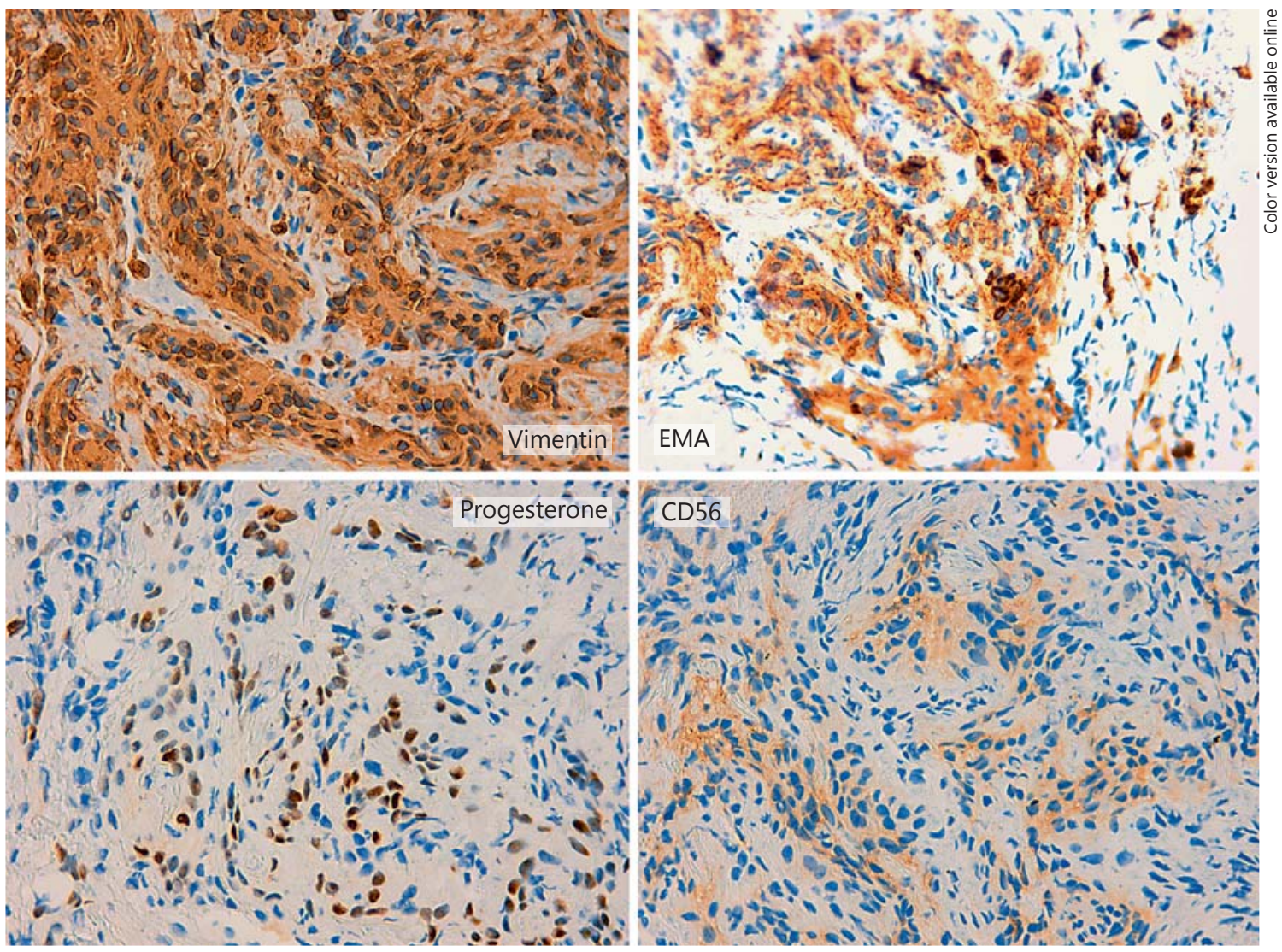

Fig. 2. Proliferation of epithelioid cells with oval monomorphic nuclei, without atypia or mitosis, arranged in irregular clusters. Immunohistochemical staining was positive for vimentin, EMA, progesterone receptors, and CD56. These findings are consistent with a diagnosis of pulmonary meningotheliomatosis.

condition is alternatively termed diffuse pulmonary meningotheliomatosis or MPMN-omatosis $[1,5,12]$. MPMNs are usually asymptomatic and are normally detected incidentally during autopsy or examination of resected lung specimens obtained for other reasons [4], whereas diffuse pulmonary meningotheliomatosis may be associated with symptoms of diffuse interstitial lung disease $[1,5]$. Diffuse pulmonary meningotheliomatosis is less common $[4,13]$; these lesions tend to affect one or several lobes of the same lung $[4,5,12]$ and can affect all the lobes of the lung, simulating metastatic disease, as in our case. Our patient also had cavitating nodules, which is a rare finding $[1,12]$ and can erroneously suggest the presence of infection or granulomatous disease. The clinical significance of MPMNs is still unclear. Mizutani et al. [13] reported a higher prevalence of these nodules in patients with malignant pulmonary tumours than in those with benign disease ( 7.3 vs. $2.5 \%$; $=0.04$ ), and also found that MPMNs were more common in patients with lung adenocarcinoma than with other types of primary lung cancers (9.4\%; odds ratio: 2.33 ; $95 \%$ confidence interval: $1.35-4.02 ; \mathrm{p}<0.01)$. Multiple MPMNs have been associated with a variety of pulmonary abnormalities: pulmonary thromboembolism $(42 \% ; p=0.01)$, respiratory bronchiolitis-associated interstitial lung disease/desquamative pneumonia (26\%; $\mathrm{p}=0.04)$, vasculitis $(24 \%)$, and atypical adenomatous hyperplasia of the lung $(12 \% ; \mathrm{p}<0.01)$ [4, 13-15]. In summary, diffuse pulmonary meningotheliomatosis should be included in the differential diagnosis of bilateral micronodular lung disease. We have described a new case of diffuse pulmonary meningotheliomatosis detected by CT and diagnosed by transbronchial lung biopsy. To our knowledge, only one case of single MPMN has been described previously in a transbronchial biopsy specimen [16]. 


\section{References}

- Kraushaar G, Ajlan AM, English JC, et al: Minute pulmonary meningothelial-like nodules: a case of incidentally detected diffuse cystic micronodules on thin-section computed tomography. J Comput Assist Tomogr 2010;34:780-782.

-2 Gaffey MJ, Mills SE, Askin FB: Minute pulmonary meningothelial-like nodules. A clinicopathologic study of so-called minute pulmonary chemodectoma. Am J Surg Pathol 1988; $12: 167-175$

- 3 Niho S, Yokose T, Nishiwaki Y, et al: Immunohistochemical and clonal analysis of minute pulmonary meningothelial-like nodules. Hum Pathol 1999;30:425-429.

4 Mukhopadhyay S, El-Zammar OA, Katzenstein ALA: Pulmonary meningothelial-like nodules: new insights into a common but poorly understood entity. Am J Surg Pathol 2009;33:487-495.

5 Suster S, Moran CA: Diffuse pulmonary meningotheliomatosis. Am J Surg Pathol 2007; $31: 624-631$.
-6 Torikata C, Mukai M: So-called minute chemodectoma of the lung. An electron microscopic and immunohistochemical study. Virchows Arch A Pathol Anat Histopathol 1990; 417:113-118.

7 Pelosi G, Maffini F, Decarli N, et al: Progesterone receptor immunoreactivity in minute meningothelioid nodules of the lung. Virchows Arch 2002;440:543-546.

8 Ionescu DN, Sasatomi E, Aldeeb D, et al: Pulmonary meningothelial-like nodules: a genotypic comparison with meningiomas. Am J Surg Pathol 2004;28:207-214.

-9 Spinelli M, Claren R, Colombi R, et al: Primary pulmonary meningioma may arise from meningothelial-like nodules. Adv Clin Pathol 2000;4:35-39.

10 Gomez-Aracil V, Mayayo E, Alvira R, et al: Fine needle aspiration cytology of primary pulmonary meningioma associated with minute meningothelial-like nodules. Acta Cytol 2002;46:899-903.

11 Kuroki M, Nakata H, Masuda T, et al: Minute pulmonary meningothelial-like nodules: highresolution computed tomography and pathologic correlations. J Thorac Imaging 2002;17: 227-229.
2 Fernández MT, Cardenal A, Furones M, et al: Diffuse pulmonary meningotheliomatosis: an uncommon cause of the micronodular pattern (in Spanish). Radiologia 2010;52:357-360.

13 Mizutani E, Tsuta K, Maeshima AM, et al: Minute pulmonary meningothelial-like nodules: clinicopathologic analysis of 121 patients. Hum Pathol 2009;40:678-682.

14 Matsuoka T, Uematsu H, Iwakiri S, et al: Minute pulmonary meningothelial-like nodules (MPMNs) incidentally detected at surgical biopsy for ground-glass opacity (GGO). Kyobu Geka 2012;65:1063-1066.

15 Kfoury H, Arafah MA, Arafah MM, et al: Mimicry of minute pulmonary meningothelial-like nodules to metastatic deposits in a patient with infiltrating lobular carcinoma: a case report and review of the literature. Korean J Pathol 2012;46:87-91.

16 Agozzino M, Inzani F, Cavallero A, et al: Minute pulmonary meningothelial-like nodules in the transbronchial biopsy of a lung transplant recipient. J Heart Lung Transplant 2006; 25:148-150. 